\title{
THE ROLE OF MAGNETIC FIELDS IN THE ORIGIN AND STRUCTURE OF PLANETARY NEBULAE
}

\author{
DONALD H. MENZEL
}

(Harvard College Observatory, Smithsonian Astrophysical Observatory, U.S.A.)

That the characteristic, quasi-symmetrical structure of planetary nebulae may result from the presence of imbedded magnetic fields has undoubtedly occurred to many astronomers. Gurzadian (1962), for example, employed the widely used equation

$$
p+H^{2} / 8 \pi=\text { const }
$$

where $p$ is the pressure of the ionized gas and $H$ the magnetic-field intensity. This equation specifies that the sum of the gas and magnetic pressures should be constant for a given value of the radius.

As often applied, however, Equation (1) is wrong. In particular it is wrong when the assumed magnetic field is a simple dipole field. For magnetic fields do not act directly on the gas. They act only on electric currents flowing in the medium. In a dipole, all the current is concentrated at or near the origin. To study this phenomenon, let us turn to the basic equation from which (1) results, under special circumstances.

The force equation of magnetohydrodynamics is:

$$
\rho \frac{\mathrm{d} \mathbf{v}}{\mathrm{d} t}=-\nabla p-\rho \nabla V+{ }_{4 \pi}^{1}(\boldsymbol{\nabla} \times \mathbf{H}) \times \mathbf{H}
$$

where $\rho$ is the density, $\mathbf{v}$ the velocity, $V$ the scalar gravitational potential, and $\mathbf{H}$ the vector magnetic field. For a dipole field the curl, $(\boldsymbol{\nabla} \times \mathbf{H})$, vanishes and thus no magnetic force exists, as previously noted. For electromagnetic forces to occur, the current density $\mathbf{J}$,

$$
\mathbf{J}=\frac{1}{4 \pi} \nabla \times \mathbf{H}
$$

must not vanish. The force is a vector, $\mathbf{J} \times \mathbf{H}$, perpendicular to both $\mathbf{J}$ and $\mathbf{H}$.

Consider a field with the following characteristics. From the vector potential,

$$
\mathbf{A}=\mu \mathbf{e}_{\theta} \frac{r \sin \theta}{\left(a^{2}+r^{2}\right)^{3 / 2}},
$$

derives the field,

$$
\mathbf{H}=\boldsymbol{\nabla} \times \mathbf{A}=\mu\left[\mathbf{e}_{r} \frac{2 \cos \theta}{\left(a^{2}+r^{2}\right)^{3 / 2}}+\mathbf{e}_{\varphi} \frac{\left(r^{2}-2 a^{2}\right) \sin \theta}{\left(a^{2}+r^{2}\right)^{5 / 2}}\right] .
$$


Here, $r, \theta, \phi$ are the usual spherical coordinates, $\mathbf{e}_{r}, \mathbf{e}_{\theta}$, and $\mathbf{e}_{\phi}$ the associated unit vectors, and $\mu$ the magnetic moment. The disposable parameter, $a$, determines the general shape of the field. When $a=0$, we obtain the characteristic dipole field with its singularity at the origin.

$$
\boldsymbol{\nabla} \times \mathbf{H}=\mu \mathbf{e}_{\phi} \frac{15 a^{2} r \sin \theta}{\left(a^{2}+r^{2}\right)^{7 / 2}} .
$$

Note that the current vanishes, as it should, for the dipole condition. For $r \gg a$, Equation (5) approximates to the standard dipole field.

Equation (2) separates, for the hydrostatic case, into two partial differential equations:

$$
\begin{gathered}
\frac{\partial p}{\partial r}=-\frac{G M \rho}{r^{2}}-\frac{15 \mu^{2} a^{2} r\left(r^{2}-2 a^{2}\right) \sin ^{2} \theta}{4 \pi\left(a^{2}+r^{2}\right)^{6}}, \\
\frac{1}{r} \frac{\partial p}{\partial \theta}=\frac{30 \mu^{2} a^{2} r \sin \theta \cos \theta}{4 \pi\left(a^{2}+r^{2}\right)^{5}} .
\end{gathered}
$$

The latter integrates immediately to

$$
p=p_{0}(r)+\frac{15 \mu^{2} a^{2} r^{2} \sin ^{2} \theta}{4 \pi\left(a^{2}+r^{2}\right)^{5}},
$$

wherein $p_{0}(r)$ is a 'constant of integration', a function of $r$ only. Clearly, it represents the pressure distribution along the polar axis, $\theta=0$ or $\pi$.

Differentiate (9) with respect to $r$, and substitute into (7). The result is:

$$
\rho=\rho_{0}+\frac{105 \mu^{2} a^{2} r^{5} \sin ^{2} \theta}{4 \pi G M\left(a^{2}+r^{2}\right)^{6}} .
$$

The second terms of (9) and (10) represent the increase of pressure and density produced by the magnetic field. The phenomenon is a true 'pinch effect', resulting from the equatorial current.

If magnetic fields are to contribute appreciably to the pressure and density, the second terms of these equations should at least be of the same order as the first. Take the extreme case where $p_{0}$ and $\rho_{0}$ are zero. The temperature distribution will then be independent of $\mu$. From the equation of state, we get

$$
T=p m / k \rho=G M m\left(a^{2}+r^{2}\right) / 7 k r^{3},
$$

where $k$ is Boltzmann's constant and $m$ the mean molecular mass.

Now let $T=10^{4}$ and $M=10^{33} \mathrm{~g}$. We get

$$
r^{3} /\left(a^{2}+r^{2}\right)=7 \times 10^{12} \mathrm{~cm} \text {. }
$$

For a planetary, we must set $r \sim 10^{17}$. Solving for $a$, we get the unreasonably large figure of $10^{19} \mathrm{~cm}$. In other words, the electric currents responsible for the field would 
have to be located in a region 100 times the radius of the luminous gas, an unacceptable condition.

The difficulty arises from the low gravitational field at large distances from the central star. No equilibrium solution is possible under such conditions, whatever the field may be, unless the temperature of the gas is reduced to the order of $1{ }^{\circ} \mathrm{K}$, when it would be neither ionized nor luminous. Introducing non-zero values for $p_{0}$ and $\rho_{0}$ does not substantially alter the problem.

This study clearly indicates that self-contained, static magnetic fields do not contribute directly to the form and structure of a planetary. Inclusion of mass motion, such as radial expansion, only serves to make matters worse, though the temperature problem no longer remains. Instead of (9), we obtain an equation something like the following:

$$
\rho v^{2} / 2=\frac{15 \mu^{2} a^{2} r^{2} \sin ^{2} \theta}{4 \pi\left(a^{2}+r^{2}\right)^{5}},
$$

in which the density of kinetic energy, with $v$ the velocity of expansion, replaces the gas pressure, $p$. But the same problem arises as before. No satisfactory simultaneous solution of (10) and (13) exists, except for physically insignificant values of $v$. Although the assumed magnetic field is somewhat arbitrary, no reasonable variation is likely to represent the problem.

Hence, although the model fails for planetary nebulae, it does hold some promise for application to magnetic stars and stars with distended atmospheres. This application will be the subject of a second paper, in which I shall develop another possible relationship between such fields and the observed structure of planetaries.

In brief, the shells that comprise a planetary nebula must be considered objects totally disconnected gravitationally or magnetically from the central star. Internal magnetic fields may still contribute to nebular structure, but no static equilibrium solution exists. For example, magnetic fields could have dictated the shell-like or ring-like structure when the nebula was very small, perhaps part of the atmosphere of the central star. After ejection, the slowly expanding shell has retained some semblance of its original form.

\section{Reference}

Gurzadian, G. A. (1962) The Magnetic Fields in Planetary Nebulae, in Vistas in Astronomy, 5, Ed. by A. Beer, Pergamon Press, New York, p. 40.

\section{DISCUSSION}

Khromov: May I ask you about the origin of the postulated magnetic field?

Menzel: If you can tell me what causes the electric currents in sunspots I can answer that question. I have postulated the existence of currents, which must occur, not just in the central star, but throughout the volume of the nebula. 